\title{
Potential Contribution of Selected Metallic Restorative Dentistry Materials to X-ray Fluorescence
}

\author{
Edson R. Andrade ${ }^{1 *}$, Ana Lucia N. Oliveira ${ }^{1,4}$, Luisa N. Funcke ${ }^{3}$, Leonardo Henrique F. F.
} Souza $^{1}$, Matthew J.F. Healy ${ }^{5}$, Helio C. Vital ${ }^{2}$

${ }^{1}$ Ibmec-RJ / Barra, Engineering Faculty, Rio de Janeiro, Brazil

${ }^{2}$ Nuclear Engineering Graduate Program, Military Institute of Engineering, Rio de Janeiro, Brazil

${ }^{3}$ Federal University of Rio de Janeiro, Chemical Engineering Faculty, Rio de Janeiro, Brazil

${ }^{4}$ Science Materials Graduate Program, Military Institute of Engineering, Rio de Janeiro, Brazil

${ }^{5}$ Cranfield Forensic Institute, Cranfield University, Defence Academy of the United Kingdom, Shrivenham, SN6 8LA, UK.

Corresponding author: Edson Ramos de Andrade, +55 21 995672184, e-mail: fisica.dna@gmail.com

\begin{abstract}
Recent advances have led to the use of new materials in dental restoration which is an area of rapid growth. Applications include improving oral aesthetics and essential rehabilitation, whilst procedures range from the recovery of partial elements (inlays) to fitting dental implants. Ceramics, polymers and metallic materials have all been successfully employed in dental applications and benefit from new cost efficient manufacturing techniques. The application of radiographic techniques in dentistry and other medicine is also increasing, and the combination of new materials and radiation can lead to an elevated health risk. X-rays can interact with metallic materials producing $\mathrm{X}$-ray fluorescence, which can increase the radiation dose in proximity to restorative material and increase the risk of live biological tissue becoming cancerous. The issue demands consideration so that the biological risks associated with such procedures are kept as low as possible. Comparisons of doses calculated for several materials have provided evidence that the Ti cp and NiCrTi alloys present less contribution to the increase of dose in surrounding soft tissue and the potential deleterious biological effects. On the other hand, Amalgam appears to be the most deleterious alloy.
\end{abstract}

Key-words: Dental; X-ray; Radiation risk; Metallic alloy 


\section{Introduction}

The use of radiological imaging techniques in modern dentistry has led to significant advances in the operational safety of X-ray machines (Wrzesien and Olszewski 2017). The proposed approach is intended to assist those who pursue producing images with minimum exposure of the patient to ionizing radiation as recommended by the ALARA (As Low As Reasonably Achievable) Principle issued by the International Commission on Radiological Protection (ICRP 2007). Many studies that address measuring and estimating exposure have been published. In addition, most of them are focused on the determination of operational exposure parameters, such as positioning, for example, and radiographic procedures and techniques and how they impact the risks associated to common dental radiographic examinations (Zenobio and da Silva 2007; Ludlow, Davies-Ludlow et al. 2008; Gavala, Donta et al. 2009). As reminded by Wrzesien and colleagues (2017), no exposure to ionizing radiation should be considered safe. Therefore, use of X-rays in dental applications must be supported by a rigorous commitment to ensure appropriate radiological protection. An important issue that has been widely discussed in Europe for over 20 years now is the fact that most of the annual radiation dose absorbed by the population and contributed by man-made sources comes from diagnostic radiology. (Schibilla and Moores 1995). Moreover, such problem affects not only developing countries but also developed ones (Faulkner, Broadhead et al. 1999).

Consequently, although much effort has been made to reduce radiation exposure of surrounding tissues in oral radiological practices over the past years, practically no attention has been given to an interesting point, which is the possibility that recently developed restorative materials can interact with the radiation field. Such interaction can emit secondary radiation according to a phenomenon known as fluorescence (Reilly and Nelson 1991).

As part of the latest advances in materials science, new compounds are increasingly being used for partial elements (inlays) and implants, in the fields of dental aesthetics and rehabilitation (Eley 1997; Allan 1999; Liedke, Spin-Neto et al. 2014) and many of them have not been fully tested . Ceramics, polymers and metallic materials are increasingly exploiting Computer-Aided Design and Manufacturing (Carrabba, Vichi et al. 2017; Otto 2017) to generate complex structures cost efficiently, and extend the utilization of novel materials through the medical sector.

Further, radiographic techniques are increasingly being used for diagnostics, and noninvasive imaging. The extent to which these new materials placed within the human body can enhance the radiation dose to live tissue and may cause harm needs to be explored. A recent study by Wrzesien and colleagues (Wrzesien and Olszewski 2017) determined the absorbed doses in brain, spinal column, thyroid and eye lens for patients, comparing different radiological procedures such as panoramic radiography, cephalometric radiography and cone beam computed tomography (CBCT). The study found that the maximum absorbed dose occurred at the brain stem for CBCT. Such an absorbed dose was about 8 times higher as compared to the average absorbed dose during panoramic radiography, and about 100 times higher than cephalometric radiography.

From the study performed by (Wrzesien and Olszewski 2017) some questions about how the different constituents materials can contribute to changes in transmission, absorption and generation of secondary radiation during the radiological sessions, which combined each other can create an additional rising spatial dose distribution. Elevating ionizing radiation doses can raise the risks of developing diseases such as cancer and the risk is going to be dependent on the radio-sensitivity of a particular organ. These additional doses from radiation interaction with the restorative materials can affect sensitive parts of the body potentially increasing the patient's detriment level. As an example, a recent study from Gang Li and colleagues (Li, Yang et al. 2018) may be addressed. Such study was aimed to monitor deleterious effects of X-ray on exfoliated buccal mucosa cells with the investigation of the dose-effect using an anthropomorphic phantom to estimate the dose, which ranged in the 0.18-3.54 mGy interval. The harm was found increased when a series of dental radiographs including a CBCT examination was performed. 
The main purpose of this work is to simulate the interaction of ionizing radiation used in dental restorative procedures considering commonly used restoration materials. The proposed approach includes sorting them according them based on the amount of secondary radiation they can produce. All simulations in this study have been intended to provide only a first qualitative idea regarding the possible contribution of such materials in the production of secondary radiation (fluorescence). Thus, this work is not aimed at quantifying such contributions as it simply disregards the geometry and composition of the facility.

Therefore, it is expected that the results from this study may be helpful in guiding appropriate selections of materials with improvements in safety to be brought by reducing the production of secondary radiation.

\section{Theory}

$\mathrm{X}$-rays can interact with materials via a range of processes including the Photoelectric Effect, which dominates over other processes at low photon energies. In this effect, the photon energy is absorbed by an atom's electron, which is immediately ejected from the atom with kinetic energy equal to that absorbed from the photon minus the energy needed to remove the electron from the atom (the ionization energy). The probability of this interaction occurring increases rapidly with atomic number, and rapidly as the energy reduces. The Equation 1 shows the photoelectric mass attenuation coefficient where $\tau$ is the photoelectric mass attenuation coefficient, $Z$ is the atomic number and $E$ is the incoming radiation energy. The experimental results show that the exponent of $Z$ actually falls into a range between 4 and 4.6 as energy varies from 0.1 Mev to $3 \mathrm{Mev}$ (Davisson and Evans 1952; Reilly and Nelson 1991). However, in the energy range explored in this study, no effects on the exponent of $Z$ are experienced.

Dental X-ray sets use photons of relatively low energy because they are sufficiently penetrating through the low $\mathrm{Z}$ material of the human body, giving sufficient image contrast to detect anomalies. However, dental restorative materials are often made of heavy metallic elements that are thus highly absorbing compared to lighter organic materials. Additionally, Dental X-ray tubes generally operate at energy ranges similar to those for general radiographic $\mathrm{X}$-ray tubes.

$$
\tau \propto \frac{Z^{4}}{E^{3}}
$$

In a stable atom, electrons occupy discrete energy levels designated by $\mathrm{K}, \mathrm{L}_{1}, \mathrm{~L}_{2}, \mathrm{~L}_{3}$, $\mathrm{M}_{1}, \ldots, \mathrm{M}_{5}$ and so forth. When an electron is removed from an inner atomic shell by any process the resulting vacancy is promptly filled by another electron from a less tightly bound shell. When K- and L-shell vacancies occur, such transition is sometimes followed by the emission of a fluorescence X-ray. The probability of this happening is called the fluorescence yield, which is a function of the atomic number for a particular atomic shell. For example, the fluorescence yield for the K-shell is seen to rise rapidly for $Z>10$, gradually approaching unity for High-Z elements. However for the same incoming photon energy the fluorescence yield for the L-shell is practically zero below copper, rising to only 0.42 at $Z=90$. The chance of a fluorescence $X-$ ray from filling the $M$ shell (or higher) is negligibly small (Reilly and Nelson 1991). Therefore, just the $\mathrm{K}$-shell has been chosen for performing the calculations.

The fluorescence yield $\left(\omega_{\mathrm{i}}\right)$, where $i$ designates the shell involved in the process, can be expressed as the ratio of the number of emitted X-rays to the total number of ionizations. Equation 2 shows the mathematical formula that approximately describes the fluorescence yield (Bertin 1975),

$$
\omega_{i}=\frac{Z^{4}}{\left(A_{i}+Z^{4}\right)}
$$


where $\mathrm{A}_{\mathrm{i}}$ is approximately $10^{6}$ for the $\mathrm{K}$ shell (Bertin 1975) and $\mathrm{Z}^{4}$ was estimated from equation (1) based on results calculated by XCOM.

\section{Methods}

The following steps were performed during this study: (a) search for metallic alloys of interest for use in dental restoration procedures; (b) photoelectric effect simulation by using XCOM (M.J. Berger 2010) for each material; (c) monitoring of the materials showing K-line excitation associated with photoelectric absorption. The program is able to generate cross sections on a standard energy grid or on a grid selected by the user and includes a combination of both. In addition, cross sections at all possible energies of for each material are automatically included in the calculations so as to provide tables and graphical displays as output (NIST 2018). The interaction coefficients were obtained from the chemical formulae entered by the user. However, it is important to notice that the cross sections for elements in the XCOM database refer to isolated neutral atoms only, not accounting for molecular and solid-state effects. The energy grid and the material chemical formula are inserted to XCOM for calculations of photoelectric (PE) coefficients on line. Equations 1 and 2 are used to estimate the fluorescence yield by assuming $\mathrm{Z}^{4}=\mathrm{E}^{3} \mathrm{x}$ PE from equation 1 and then calculating from equation 2.

Metal alloys are common in dental restoration procedures. Because they are a mixture of metals, in comparison with lighter elements they may contribute to an even more significant risk as the photoelectric emission rises, according to eq.1. These alloys have an effective $Z$ value which may vary from the sum of the individual atomic numbers in the mixture depending on how the average is calculated (Murty 1965; M.J. Berger 2010), increasing the photoelectric effect absorption, and consequently fluorescence yield. Because the energy range for dental applications is narrow, the energy interval considered for the simulations ranged from 10 to 100 $\mathrm{keV}(0.1 \mathrm{MeV})$. However, a wider range reaching $\mathrm{MeV}$ energies would be welcome as CBCT examination or even radiotherapy ordinarily is performed in such energy levels.

\section{Results}

The effective atomic number (Zeff), the fluorescence X-rays yield and the photoelectric absorption coefficients were estimated from eq. 1 and 2, as appropriate. Table 1 shows the main features of interest for each material tested. The alloys are listed in decreasing order of density, where the atomic number of each element is included in brackets.

\section{TABLE 1 NEAR HERE}

The Figure 1 shows the Effective atomic number (Zeff) calculated according to the methodology developed by Murty and colleagues (Murty 1965).

\section{FIGURE 1 NEAR HERE}

The Figure 2 shows, respectively, the photoelectric absorption (PE) calculation (2A) and the X-ray fluorescence yield estimate profile (2B) for the materials listed in Table 1 accordingly to equations 1 and 2 .

\section{FIGURE 2 NEAR HERE}

\section{Discussion}

Figure 1 shows that the differences between the effective atomic numbers (Zeff) of the molecules constituting the alloys remain within the same range of values, except for the amalgam, which shows Zeff around 3 times the average Zeff for the others. This characteristic 
allows inferring toward the higher production of secondary photons in comparison to the all other alloys of interest for this study.

The Figure 2(A and $\mathrm{B}$ ) shows the results for all metal alloys concerning to the photoelectric effect and fluyorescence yield for energies up to $0.1 \mathrm{MeV}(100 \mathrm{keV})$. The results are in agreement with the theory and suggest that the phenomenon has its probability of occurrence in direct proportionality with Zeff and inversely proportional to the photons' energy.

A comparison between the results shown in Figures 2A and 2B suggests that the higher the photoelectric absorption (PE) is, the lower the contribution of the material to the production of X-ray fluorescence becomes. Such trend is in agreement with the theory, since photons participating in the photoelectric absorption phenomenon are mostly removed from the primary radiation beam and do not contribute to the fluorescence process (Reilly and Nelson 1991). As suggested from the results shown in figures $2 \mathrm{~A}$ and $2 \mathrm{~B}$, the alloys with the highest photoelectric absorption coefficients, such as $\mathrm{Ti} \mathrm{cp}$ and $\mathrm{NiCrTi}$, are the ones that contribute least to the emission of $\mathrm{X}$ radiation by fluorescence processes. In contrast, Amalgam, which presents virtually no photoelectric absorption, has high estimated values of X-ray fluorescence production, and it is reasonable to suppose that it yields greater contributions to the radiation doses absorbed by the patient undergoing radiological examinations for dental treatments.

Considering the metallic materials under appreciation, it can be verified from the simulation that NiCrTiis the material that less contributes to the production of secondary X-rays by fluorescence. On the other hand, the one that contributes the most is the Amalgam. It should be noticed for all alloys under study that the dependency on energy follows the same pattern. Therefore, considering both results found in the simulations an interesting issue to be checked rises. Studying radiological behavior of materials used in dental restoration can potentially affect Public Health strategies by changing materials used as dental restoration in order to reduce risks by reducing radiation exposure. From these simulations and results Amalgam, when compared to the other tested materials, seems to increase secondary $\mathrm{X}$ radiation in dental radiological procedures. Such a behavior might lead to a potential negative impact to the risk of detriment to the patient by the increasingly profile of producing secondary radiation. Many groups have been increasingly investigating this secondary radiation profile lately. However, such studies are mostly focused on therapy instead of the diagnostic use of radiation (Ludlow, Davies-Ludlow et al. 2008; Mesbahi, Seyednejad et al. 2010; Rehman, Isa et al. 2018). Nevertheless, this increase in the scattered X-rays by restorative materials in dental care can effectively alter the interaction with the surrounding soft tissues, by producing additive effects on the dose of absorbed radiation by them. In addition, this behavior may lead to an increase in the potential risks of developing deleterious effects not only in the mouth region but also nearby as far the radiation field is able to reach sensible tissues and organs surrounding.

\section{Conclusion}

The use of metallic materials as restorative elements in dental procedures associated with technologies involving ionizing radiation is increasing. This trend requires additional care so that risks associated with such procedures are not increased. Thus, the issue of scattered Xray fluorescence being produced due to the interaction of the primary X-ray beam with recently developed materials used for dental use indicates that there is a need to define criteria for the choice of such materials. A comparison among tested materials indicates that the Ti $\mathrm{cp}$ and $\mathrm{NiCrTi}$ alloys result in the smaller contribution to the absorbed doses by surrounding soft tissues with less impact to potential deleterious effects to the patients undergoing radiological procedures. On the other hand, Amalgam appear to be the most deleterious alloy.

Furthermore, the doses from panoramic radiography and cephalometric radiography exams can be considered to be low compared to other imaging modalities such as and cone beam computed tomography (CBCT). However, such doses can be raised by repeating the exams many times getting even risky if a patient has amalgam implants due to the potentially raised secondary radiation. Moreover, a simply public health strategy against the potential increased radiological risk could be in the sense of encouraging the exchange of Amalgam by other alloys less likely to emit secondary radiation in dental restoration procedures. 


\section{Conflicts of interest}

No conflict of interest.

\section{Acknowledgements}

The authors wish to thank the colleagues who have diligently contributed with many useful comments and suggestions. We also thank the Ibmec-RJ/Barra Faculty of Engineering for the technical and material support.

\section{References}

Allan, D. N. (1999). "Dental restoration review praised." Br Dent J 187(12): 632.

Bertin, E. P. (1975). Principles and Practice of X-Ray Spectrometric Analysis. New York, Plenum Press.

Carrabba, M., A. Vichi, et al. (2017). "Comparison of traditional and simplified methods for repairing CAD/CAM feldspathic ceramics." J Adv Prosthodont 9(4): 257-264.

Davisson, C. M. and R. D. Evans (1952). "Gamma-Ray Absorption Coefficients." Reviews of Modern Physics 24(2): 79-107.

Eley, B. M. (1997). "The future of dental amalgam: a review of the literature. Part 7: Possible alternative materials to amalgam for the restoration of posterior teeth." $\mathrm{Br}$ Dent $\mathrm{J}$ 183(1): 11-14.

Faulkner, K., D. A. Broadhead, et al. (1999). "Patient dosimetry measurement methods." Appl Radiat Isot 50(1): 113-123.

Gavala, S., C. Donta, et al. (2009). "Radiation dose reduction in direct digital panoramic radiography." Eur J Radiol 71(1): 42-48.

ICRP (2007). "ICRP Publication 105. Radiation protection in medicine." Ann ICRP 37(6): 1-63.

Li, G., P. Yang, et al. (2018). "Buccal mucosa cell damage in individuals following dental X-ray examinations." Sci Rep 8(1): 2509.

Liedke, G. S., R. Spin-Neto, et al. (2014). "Radiographic diagnosis of dental restoration misfit: a systematic review." J Oral Rehabil 41(12): 957-967.

Ludlow, J. B., L. E. Davies-Ludlow, et al. (2008). "Patient risk related to common dental radiographic examinations: the impact of 2007 International Commission on Radiological Protection recommendations regarding dose calculation." J Am Dent Assoc 139(9): 1237-1243.

M.J. Berger, J. H. H., S.M. Seltzer, J. Chang, J.S. Coursey, R. Sukumar, D.S. Zucker, and K. Olsen (2010). XCOM: Photon Cross Sections Database.

Mesbahi, A., F. Seyednejad, et al. (2010). "Estimation of organs doses and radiation-induced secondary cancer risk from scattered photons for conventional radiation therapy of nasopharynx: a Monte Carlo study." Jpn J Radiol 28(5): 398-403.

Murty, R. C. (1965). "Effective Atomic Numbers of Heterogeneous Materials." Nature 207: 398.

NIST. (2018). "How to Run the XCOM Program." Retrieved 16 May 2018, 2018, from https://physics.nist.gov/PhysRefData/Xcom/Text/intro.html.

Otto, T. (2017). "Up to 27-years clinical long-term results of chairside Cerec 1 CAD/CAM inlays and onlays." Int J Comput Dent 20(3): 315-329.

Rehman, J. U., M. Isa, et al. (2018). "Dosimetric, Radiobiological and Secondary Cancer Risk Evaluation in Head-and-Neck Three-dimensional Conformal Radiation Therapy, Intensity-Modulated Radiation Therapy, and Volumetric Modulated Arc Therapy: A Phantom Study." J Med Phys 43(2): 129-135.

Reilly, D. and G. Nelson (1991). Gamma-Ray Interactions with Matter. Passive Nondestructive Assay of Nuclear Materials. S. Kreiner. Washington, DC 20555, Los Alamos National Laboratory: $27-42$. 
Schibilla, H. and B. M. Moores (1995). "Diagnostic radiology better images--lower dose compromise or correlation? A European strategy with historical overview." J Belge Radiol 78(6): 382-387.

Wrzesien, M. and J. Olszewski (2017). "Absorbed doses for patients undergoing panoramic radiography, cephalometric radiography and CBCT." Int J Occup Med Environ Health 30(5): 705-713.

Zenobio, M. A. and T. A. da Silva (2007). "Absorbed doses on patients undergoing tomographic exams for pre-surgery planning of dental implants." Appl Radiat Isot 65(6): 708-711.

Table 1 - Main features of each alloy tested as selected materials

\begin{tabular}{|c|c|c|}
\hline Alloy & $\begin{array}{r}\text { Density } \\
\left(\mathrm{g} / \mathrm{cm}^{3}\right)\end{array}$ & Weighted Composition (\%) \\
\hline Amalgam & 9.0 & $\mathrm{Hg}(46), \mathrm{Ag}(29), \mathrm{Sn}(17), \mathrm{Cu}(7), \mathrm{Zn}(1)$ \\
\hline CoCrMoW $\left(\right.$ Remanium $^{\circledR} 2000^{+}$) & 8.6 & Co (63), Cr (23), Mo (7), W (5), Si (1.5) \\
\hline CoCrMo $\left(\right.$ Biosil $^{\circledR}$ F CE 0124) & 8.4 & Co (64.8), Cr (28.5), Mo (5.3), Si (0.5), Mn (0.5), C (0.4) \\
\hline $\mathrm{NiCr}(\mathrm{HB} 28)$ & 8.0 & $\mathrm{Ni}$ (77.0), $\mathrm{Cr}$ (13.7), $\mathrm{Mo}$ (3.1), Ti (0.28), Nb (2.60), Al (2.45) \\
\hline NiCrTi (Tilite ${ }^{\circledR}$ Omega) & 7.7 & $\mathrm{Ni}$ (72.0), Cr (17.0), Ti (6.0), Mo (4.5), Be (0.5) \\
\hline Ti cp (Tritan) & 4.5 & $\mathrm{Ti}$ (99.5), N (0.05), C (0.08), H (0.015), Fe (0.2), O (0.145) \\
\hline Ti6Al4V & 4.4 & $\begin{array}{l}\mathrm{Ti}(89.57), \mathrm{N}(0.02), \mathrm{C}(0.01), \mathrm{H}(0.003), \mathrm{Fe}(0.22), \mathrm{O}(0.17), \mathrm{Al} \\
(6.20), \mathrm{V}(3.80)\end{array}$ \\
\hline
\end{tabular}

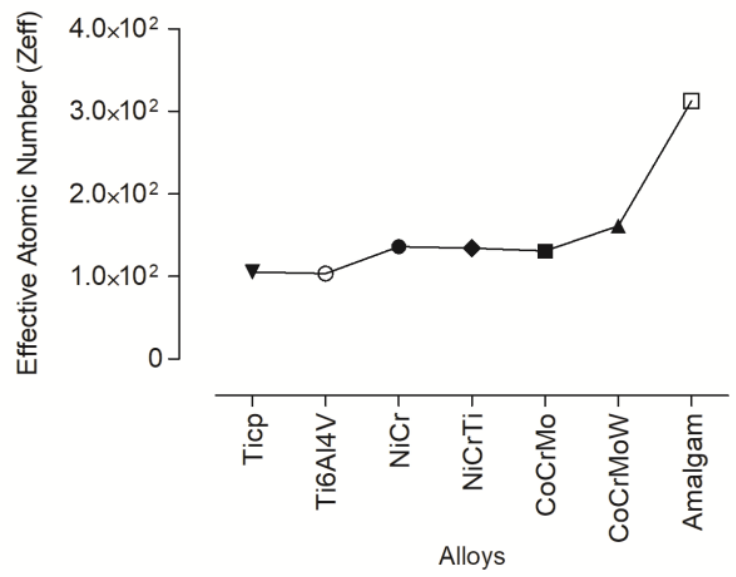

Figure 1 shows the Effective atomic number (Zeff). 

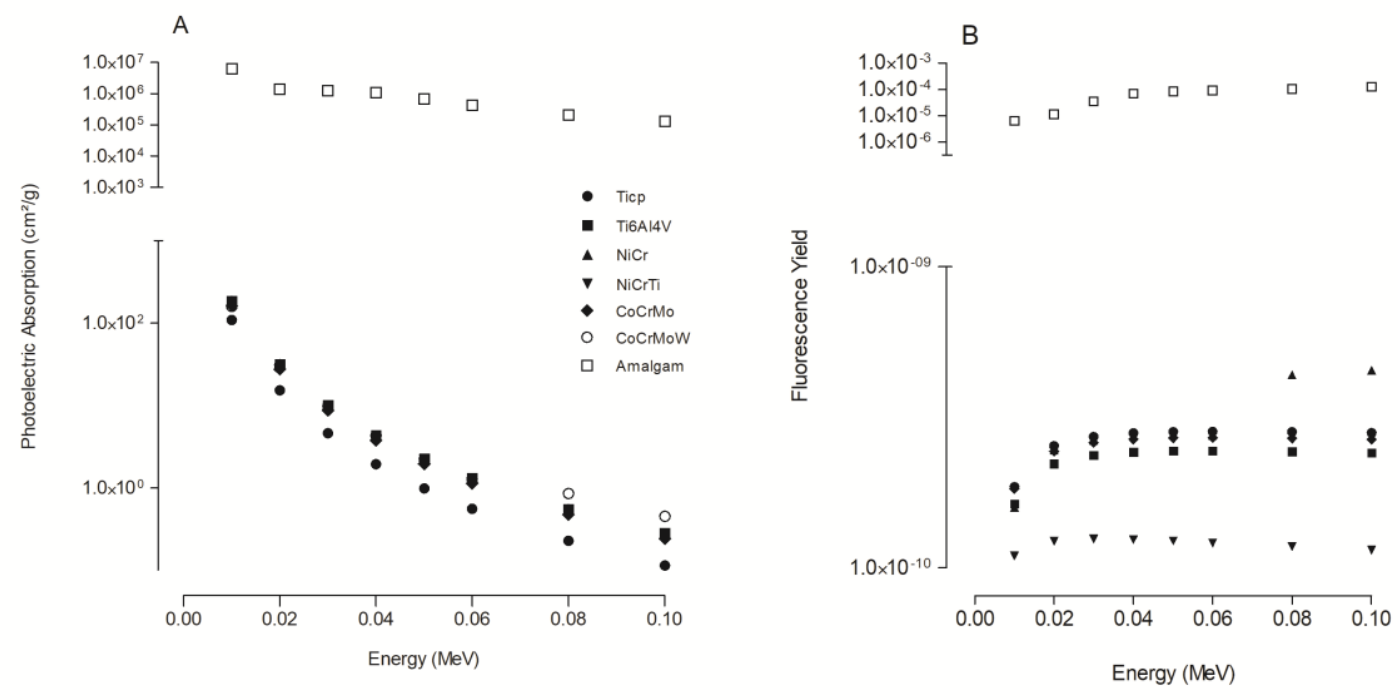

Figure 2. The photoelectric absorption (PE) calculation (2A) and the X-ray fluorescence yield estimate profile (2B) for the materials listed in Table 1 accordingly to equations 1 and 2. 